\title{
Functional Characterization of the C-Terminus of YhaV in the Escherichia coli PrIF-YhaV Toxin-Antitoxin System ${ }^{\mathrm{S}}$
}

\author{
Wonho Choi ${ }^{1,2}$, Min-Ho Yoon ${ }^{2 *}$, and Jung-Ho Park ${ }^{1 *}$ \\ ${ }^{1}$ Bio-Evaluation Center, Korea Research Institute of Bioscience and Biotechnology, Cheongju 28116, Republic of Korea \\ ${ }^{2}$ Department of Bio-Environmental Chemistry, College of Agriculture and Life Sciences, Chungnam National University, Daejeon 34134, \\ Republic of Korea
}

\author{
Received: March 8, 2018 \\ Revised: April 1, 2018 \\ Accepted: April 5, 2018 \\ First published online \\ April 12, 2018 \\ ${ }^{*}$ Corresponding authors \\ J.-H.P. \\ Phone: +82-43-240-6547; \\ Fax: +82-43-240-6549; \\ E-mail: jungho@kribb.re.kr \\ M.-H.Y. \\ Phone: +82-42-821-6733; \\ Fax: +82-42-823-9241; \\ E-mail: mhyoon@cnu.ac.kr \\ S upplementary data for this \\ paper are available on-line only at \\ http://jmb.or.kr. \\ pISSN 1017-7825, eISSN 1738-8872 \\ Copyright(C) 2018 by \\ The Korean Society for Microbiology \\ and Biotechnology
}

Bacterial programmed cell death is regulated by the toxin-antitoxin (TA) system. YhaV (toxin) and Pr1F (antitoxin) have been recently identified as a type II TA system in Escherichia coli. YhaV homologs have conserved active residues within the $\mathrm{C}$-terminus, and to characterize the function of this region, we purified native $\mathrm{YhaV}$ protein (without denaturing) and constructed YhaV proteins of varying lengths. Here, we report a new low-temperature method of purifying native $\mathrm{YhaV}$, which is notable given the existing challenges of purifying this highly toxic protein. The secondary structures and thermostability of the purified native protein were characterized and no significant structural destruction was observed, suggesting that the observed inhibition of cell growth in vivo was not the result of structural protein damage. However, it has been reported that excessive levels of protein expression may result in protein misfolding and changes in cell growth and mRNA stability. To exclude this possibility, we used an $\left[{ }^{35} \mathrm{~S}\right]$-methionine prokaryotic cell-free protein synthesis system in vitro in the presence of purified $\mathrm{YhaV}$, and two C-terminal truncated forms of this protein (YhaV-L and YhaV-S). Our results suggest that the YhaV C-terminal region is essential for mRNA interferase activity, and the W143 or H154 residues may play an analogous role to Y87 of RelE.

Keywords: Toxin-antitoxin system, YhaV, PrlF, truncation, circular dichroism, Escherichia coli

\section{Introduction}

Most bacteria have a toxin-antitoxin system (TA) that regulates cell growth and death in response to various stressful conditions [1, 2]. To date, six types of TA systems have been characterized according to how antitoxins regulate the function of their cognate toxins [3]. An antisense RNA antitoxin can regulate a toxin's translation by binding to the mRNA of its cognate toxin (type I) [4-6] or to the toxin protein directly (type III). The type I system is composed of an mRNA antitoxin and a protein toxin. Hok-Sok, LdrD-RdID, and SymE-SymR were reported as type I TA systems. In type IV TA systems, an antitoxin does not bind to a toxin, but this antitoxin has the opposite function of its related toxin. In type $\mathrm{V}$, an antitoxin protein has RNase activity that is specific to its cognate toxin, and an antitoxin in type VI induces the degradation of its toxin as an adapter protein between a toxin and a protease. The type II systems are the most studied TA system and are divided into two groups on the basis of their ribosome dependency for their enzymatic activity; ribosomedependent or ribosome-independent [1]. Among them, toxins that cleave mRNA and inhibit cell growth are called mRNA interferases [1]. This system is composed of two proteins, a stable toxin and an unstable antitoxin capable of neutralizing toxins. When bacteria are under stress, unstable antitoxins are rapidly degraded by proteases (e.g., an ATPdependent protease, Lon, or ClpX family) [7]. Therefore, toxins of TA systems bind to DNA, RNA, or protein targets and inhibit cell growth through various mechanisms, including multidrug tolerance, biofilm formation, and cell persistence mechanisms [8-10]. In the E. coli MazEF TA system, the MazF toxin is a ribosome-independent mRNA interferase that inhibits bacterial cell growth by cleaving 
mRNA at $\mathrm{A}^{\wedge} \mathrm{CA}(\wedge$ indicates the cleavage site) [11]. In addition, the tertiary structures of MazEF indicate that MazF forms a heterohexamer complex with the antitoxin MazE $\left(\mathrm{MazF}_{2}-\mathrm{MazE}_{2}-\mathrm{MazF}_{2}\right)$ [12]. Another ribosomeindependent TA system of E. coli, MqsR-MqsA, involves the toxin MqsR, which induces the inhibition of cell growth by mRNA cleavage at GCU or GUA [13]. Compared with ribosome-independent mRNA interferases, the target mRNA recognition sites of ribosome-dependent mRNA interferases are relatively short and most are codonrelated. One well-characterized ribosome-dependent mRNA interferase, the RelE toxin in the RelBE TA system, binds to the A site of the ribosome and cleaves mRNAs at $\mathrm{UA}^{\wedge} \mathrm{G}$ and $\mathrm{UA}^{\wedge} \wedge \mathrm{A}$ sequences of termination codons, leading to the inhibition of cell growth [14-16]. An NMR study that analyzed the structure of the toxin RelE indicated that the endoribonuclease active site locates primarily to the C-terminus [17]. Furthermore, other ribosome-dependent TA systems, YoeB-YefM [18], HipA-HipB [19], and YafOYafN [20], are also reported to bind to the ribosomes of E. coli $30 \mathrm{~S}$ or $50 \mathrm{~S}$ ribosomal subunits and have their mRNA interferase activities as toxin-ribosome-mRNA substrate $[14,21]$. Previously, PrlF-YhaV was classified as a type II TA system, based in part on homology to the ribosomedependent YoeB and RelE families [22]. Ribosome profiling analysis of the toxin $\mathrm{YhaV}$ revealed that the expressed toxin YhaV bound to the $50 \mathrm{~S}$ ribosomal subunit and inhibited bacterial growth [23]. The RelE toxin is a well-known global inhibitor of translation, and structural analysis suggests that the active site of RelE toxin is located in the C-terminal amino acid region $[24,25]$.
Here, to evaluate the function of the C-terminus of YhaV, which is a conserved region in $\mathrm{YhaV}$ homologs, we purified the native $\mathrm{YhaV}$ protein and C-terminal truncated proteins of various lengths (without denaturing) for the first time. By determining the secondary structures and enzyme activities of full-length $\mathrm{YhaV}$ and various C-terminal truncated forms, we show that the YhaV C-terminal residues from 131 to 145 are involved in mRNA interferase activity.

\section{Materials and Methods}

\section{Bacterial Strains, Plasmids, and Primers}

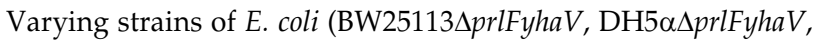
and BL21(DE3) $\Delta$ prlFyhaV) were used in this analysis [23]. Various versions of YhaV (yhaV $241-465, \Delta 271-465, \Delta 301-465, \Delta 331-465$, $\Delta 361-465, \Delta 391-465, \Delta 406-465, \Delta 421-465, \Delta 436-465$, or $\Delta 451-465)$ were amplified by PCR using pBAD24-yhaV as the template with the primers listed in Table 1. These fragments and $\mathrm{pBAD}$ vector were digested by NdeI and XhoI DNA restriction enzymes. Purified inserts were then cloned into the pBAD24 vector with DNA ligase [23]. The yhaV $4336-465$ encoding YhaV-L and $\Delta 391-$ 465 encoding YhaV-S genes were also separately cloned into the pET28 vector (Novagen, USA) to express the C-terminal truncated YhaV proteins, following the protocol as described above. All constructs were confirmed by DNA sequencing. All the primers used in this study are listed in Table 1.

\section{Measuring the Toxicity of $\mathrm{YhaV}$ and C-terminal Truncated Forms of YhaV

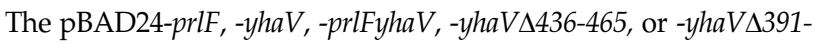

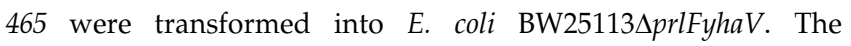 transformants were streaked onto M9 agar plates in the presence or absence of $0.2 \%$ arabinose and grown at $37^{\circ} \mathrm{C}$. The growth}

Table 1. Primer designations and sequences.

\begin{tabular}{|c|c|}
\hline Primer & Sequence \\
\hline YhaV-F & 5'-TATACATATGGATTTTCCACAAAGGGTTA-3' \\
\hline $\mathrm{YhaV}(\Delta 81-154)-\mathrm{R}$ & 5'-TATACTCGAGTCAATTTTTCCCAGAGCCT-3' \\
\hline YhaV( $\Delta 91-154)-\mathrm{R}$ & 5'-TATACTCGAGTCAAGCACCAAATTTTACC-3' \\
\hline YhaV( $\Delta 101-154)-\mathrm{R}$ & 5'-TATACTCGAGTCAACTATAACGAAAGAAG-3' \\
\hline YhaV( $\Delta 111-154)-\mathrm{R}$ & 5'-TATACTCGAGTCACCATCCCAGAATGATG-3' \\
\hline YhaV( $\Delta 121-154)-\mathrm{R}$ & 5'-TATACTCGAGTCAGTAGGTGCGCAGAGTG-3' \\
\hline YhaV( $\Delta 131-154)-\mathrm{R}$ & 5'-TATACTCGAGTCAGAATACGGTATAGGCA-3' \\
\hline YhaV( $\Delta 136-154)-\mathrm{R}$ & 5'-TATACTCGAGTCATTTTAACATTTTGCTG-3' \\
\hline YhaV( $\Delta 141-154)-\mathrm{R}$ & 5'-TATACTCGAGTCAAGGAGGATGTCCTCTT-3' \\
\hline YhaV( $\Delta 146-154)-\mathrm{R}$ & 5'-TATACTCGAGTCAGGTTTCCCAGTCGGCA-3' \\
\hline YhaV( $\Delta 151-154)-\mathrm{R}$ & 5'-TATACTCGAGTCATGTTTCTCGGGTGAGG-3' \\
\hline ompF-F & 5'-CTTTGGTCTGGTTGATGGCC-3' \\
\hline ompF-R & 5'-ACTTCAGACCAGTAGCCCAC-3' \\
\hline
\end{tabular}


curves were measured using E. coli BW25113 $\Delta$ prlFyhaV cells harboring pBAD24, pBAD24-yhaV, -yhaVA436-465, or -yhaV 391 465 in M9 liquid medium at $37^{\circ} \mathrm{C}$ in the presence of $0.2 \%$ arabinose.

\section{RNA Isolation and Northern Blot Analysis}

E. coli BW25113 $\mathrm{prlFyhaV}$ cells harboring pBAD24, pBAD24$y h a V$, $-y h a V \Delta 436-465$, or $-y h a V \Delta 391-465$ were cultured in M9 medium with $0.2 \%$ glycerol at $37^{\circ} \mathrm{C}$. When the culture's $\mathrm{OD}_{600}$ value reached 0.4 , the expression of $\mathrm{YhaV}$, YhaV-L, or YhaV-S was induced by adding arabinose to a final concentration of $0.2 \%$. Aliquots of cell cultures were taken at $0,1,3$, and $6 \mathrm{~h}$. Total RNA was extracted by using Trizol (Thermo Fisher Scientific, USA) following the manufacturer's protocol. Northern blot analysis was carried out as previously described with modified probes, ompF-F and ompF-R, that were labeled with biotin-14-dCTP [26]. All probes used in this study are shown in Table 1.

\section{Protein Expression and Purification}

To express and purify YhaV, YhaV-L, and YhaV-S, pET28-yhaV, -yhaV $\Delta 436-465$, and -yhaV $391-465$ were transformed into E. coli BL21(DE3) $\Delta$ prlFyhaV. When the $\mathrm{OD}_{600}$ value of the cultures reached 0.5, 0.5 mM isopropyl- $\beta$-D-1-thiogalactoside was added to induce YhaV, YhaV-L, and YhaV-S protein expression. The cultures were incubated at $18^{\circ} \mathrm{C}$ for $6 \mathrm{~h}$. N-Terminally $\mathrm{His}_{6}$-tagged YhaV, YhaV-L, and YhaV-S were purified using Ni-NTA-agarose (Qiagen, Germany) following the manufacturer's protocol. Additionally, C-terminally $\mathrm{His}_{6}$-tagged $\mathrm{YhaV}$ was purified with Ni-NTA-agarose followed by the solid-phase refolding method with $6 \mathrm{M}$ guanidine- $\mathrm{HCl}$ from cells harboring pColdIII-prlFyhaV. All purified proteins were analyzed by SDS-PAGE (sodium dodecyl sulfate polyacrylamide gel electrophoresis) followed by Coomassie stain. For western blot analysis, purified proteins were resolved on $10 \%$ SDS-polyacrylamide gels and transferred to polyvinylidene difluoride membranes. The blots were probed with a polyclonal anti-histidine tag antibody (\#2365; Cell Signaling Technology, USA).

\section{Circular Dichroism (CD) Analysis of YhaV and C-Terminal Truncated Forms of YhaV}

Far-UV CD measurements were carried out using an automated Chirascan CD spectrometer (Applied Photophysics, UK). The spectra were recorded over a wavelength range of 190-260 nm using a $0.5-\mathrm{mm}$ path length from $25^{\circ} \mathrm{C}$ to $85^{\circ} \mathrm{C}$ at $10^{\circ} \mathrm{C}$ intervals to determine the secondary structure of YhaV, YhaV-L, and YhaV-S. To determine the melting points of $\mathrm{YhaV}, \mathrm{YhaV}-\mathrm{L}$, and $\mathrm{YhaV}-\mathrm{S}$, the temperature was increased by $0.2^{\circ} \mathrm{C}$ stepwise from $25^{\circ} \mathrm{C}$ to $90^{\circ} \mathrm{C}$. Purified proteins were desalted by dialysis solution equilibrated with $10 \mathrm{mM}$ sodium phosphate $(\mathrm{pH} 7.8)$, and then adjusted to $0.3 \mathrm{mg} / \mathrm{ml}$ protein concentration for $\mathrm{CD}$ analysis. The background $\mathrm{CD}$ spectrum of the buffer was subtracted from the $\mathrm{CD}$ spectra of the protein samples.

Effect of C-Terminal Truncated Forms of $\mathrm{YhaV}$ on Protein Synthesis in Prokaryotic Cell-Free Systems

A prokaryotic cell-free protein synthesis assay was performed with the E. coli T7 S30 extract system (Promega, USA), following the manufacturer's protocol. Different concentrations (100, 500, and 1,000 nM) of YhaV, YhaV-L, and YhaV-S were added to the reaction mixtures, following a previous protocol [23]. The pellets were analyzed by SDS-PAGE followed by autoradiography.

\section{Results}

The Sequence of YhaV Is Highly Similar to RibosomeDependent mRNA Interferases Harboring Active Residues in the C-Terminal Region

The prlF-yhaV operon is located at $70.59 \mathrm{~min}$ on the E. coli K12 chromosome. YhaV and PrlF have 154 and 111
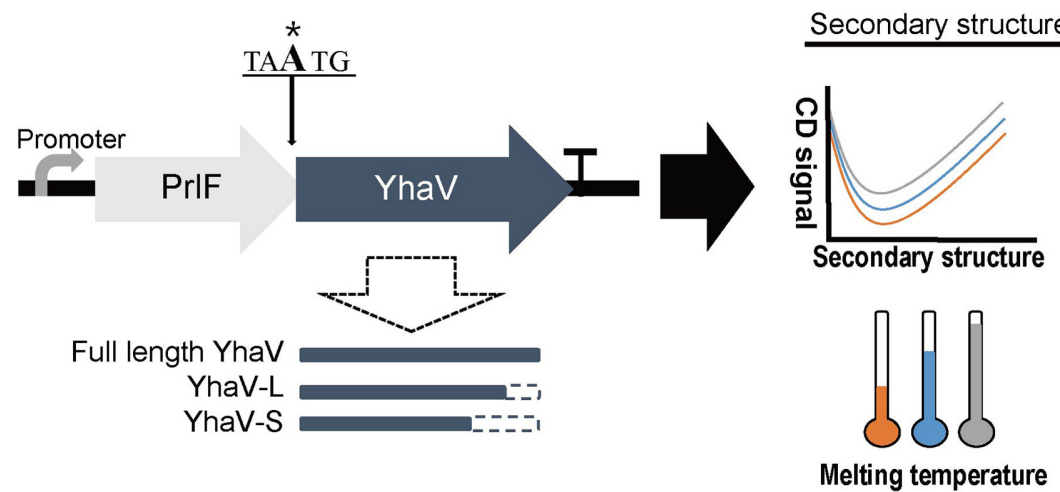

Growth inhibition
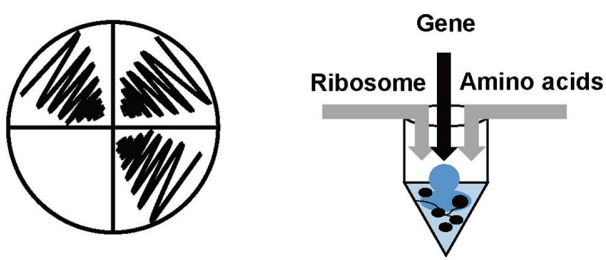

Enzyme activity assay

Fig. 1. Schematic diagram of the study constructs and protocols.

To characterize the function of the C-terminal region of YhaV, C-terminal truncated forms of YhaV were generated. The secondary structures of the C-terminal truncated forms of YhaV were characterized using circular dichroism. The functions of YhaV's C-terminal region were evaluated using in vivo toxicity and in vitro translation assays. 
amino acid residues, respectively. Both prlF and yhaV are located in the same operon, and the stop codon of $p r l F$ and the start codon of yhaV overlap by one base (Fig. 1). In a previous report, YhaV was aligned to other toxin proteins with known tertiary structures to determine their similarity. With the notable exception of a 20-residue insertion between the first and second $\alpha$-helices in $\mathrm{YhaV}$, high homology between YhaV and YoeB and RelE from E. coli and RelE from Pyrococcus horikoshii was observed [22]. To identify the mRNA interferase activity region, we aligned the amino acid sequences of ribosome-dependent mRNA interferases RelE (PDB ID: 4FXI; Chain A), YoeB (PDB ID: 2A6Q; Chain E), and HigB (PDB ID: 4ZSN; Chain QY or $\mathrm{XY}$ ) using T-Coffee, a multiple sequence alignment tool (Fig. 2A) [12, 27-33]. Using this approach, we observed that ribosome-dependent mRNA interferases were structurally similar (Fig. 2A). Moreover, tertiary structure alignments were carried out with the magic fit algorithm, a sequencebased structural alignment tool from Swiss-PDB Viewer ver. 4.1.0, and imaged in Jmol 14.6.5 [34, 35]. Interestingly, the secondary structure of MqsR, known as ribosomeindependent (PDB ID: 3HI2; Chain B), was similar to

A

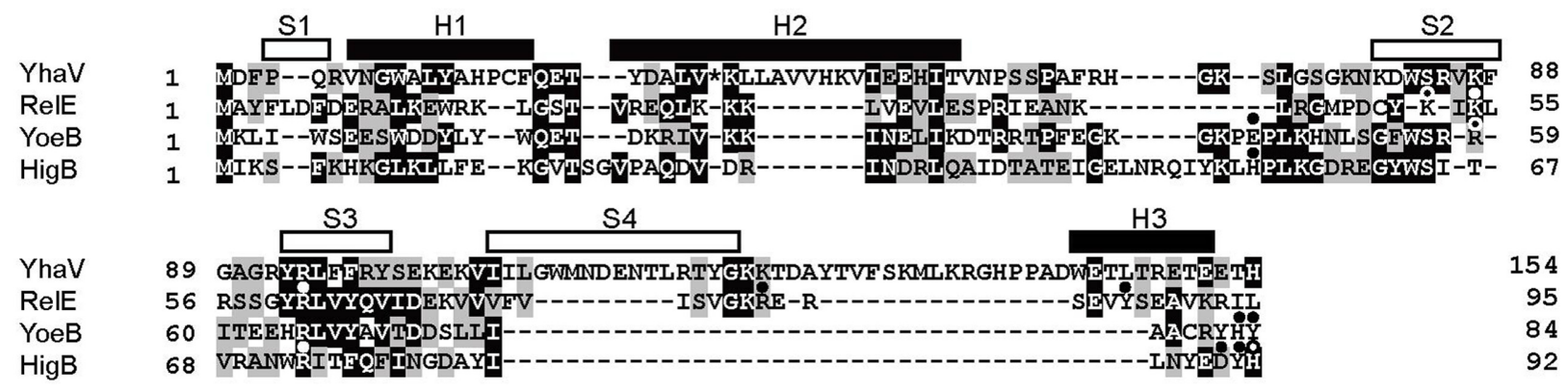

B

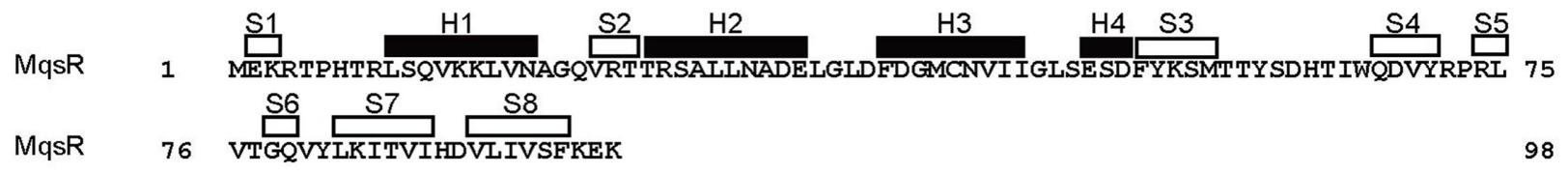

C
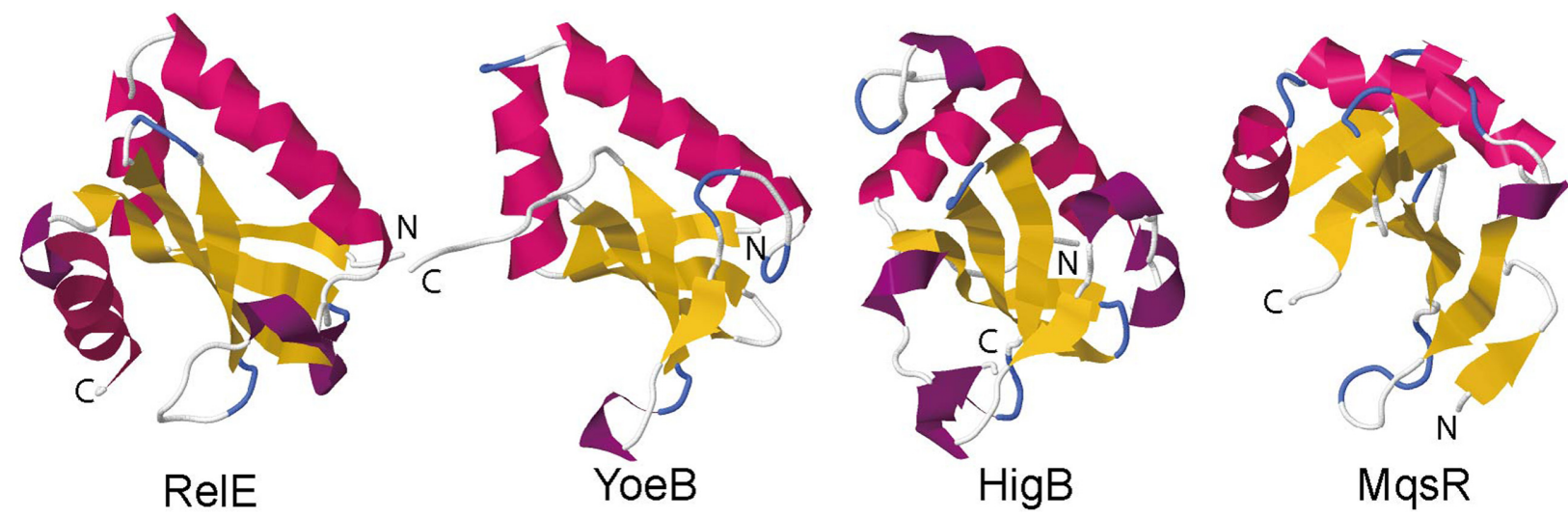

Fig. 2. Sequence alignments and tertiary structure comparisons of $\mathrm{YhaV}$ and other TA system toxins.

(A) Multiple sequence alignments of YhaV and ribosome-dependent toxins (RelE (PDB ID: 4FXI; Chain A), YoeB (PDB ID: 2A6Q; Chain E) and HigB (PDB ID: 4ZSN; Chain QY or XY)). White and black boxes indicate $\beta$-sheets (S) and $\alpha$-helices (H), respectively, on the basis of the secondary structures of RelE (PDB ID: 4FXI). The asterisk shows 20 elliptical residues, AEVETLKGKDPENYQRKAAT. Black circles show active site residues. Similar residues having threshholds of more than 50\% in the BLOSUM62 matrix are shaded grey and identical residues are shaded black. (B) Secondary structure of the ribosome-independent toxin MqsR (PDB ID: 3HI2; Chain B). White and black boxes indicate $\beta$-sheets (S) and $\alpha$-helices $(\mathrm{H})$, respectively. (C) Comparison of the tertiary structures of four TA system toxins. Tertiary structure alignments were carried out with magic fit, a sequence-based structural alignment tool from Swiss-PDB Viewer ver. 4.1.0, among RelE (PDB ID: 4FXI; Chain A), YoeB (PDB ID: 2A6Q; Chain E), HigB (PDB ID: 4PX8; Chain A), and MqsR (PDB ID: 3HI2; Chain B). All structures were imaged in Jmol 14.6.5. The N-terminal and C-terminal ends of the tertiary structures are represented by $N$ and $C$, respectively. 
ribosome-dependent mRNA interferases in the $\mathrm{N}$-terminal region, which has a small $\beta$-sheet followed by two $\alpha$ helices. The tertiary structure of MqsR, however, was quite different than the three ribosome-dependent toxins, RelE, YoeB, and HigA (Figs. 2B and 2C) [36]. It is suggested that $\mathrm{YhaV}$, similar to other ribosome-dependent mRNA interferases, also has catalytic residues in the C-terminal region.

\section{YhaV-S Have Reduced Toxicity Compared with Native YhaV and YhaV-L}

As the tertiary structure of YhaV has not been reported, we generated $\mathrm{YhaV}$ variants with various C-terminal truncations to identify active residues. The cell toxicity effects of $10 \mathrm{C}$-terminal truncated forms of $\mathrm{YhaV}$ were quantified by culturing cells transformed with these constructs on plates at $24^{\circ} \mathrm{C}, 37^{\circ} \mathrm{C}$, or $42^{\circ} \mathrm{C}$ in the presence

A

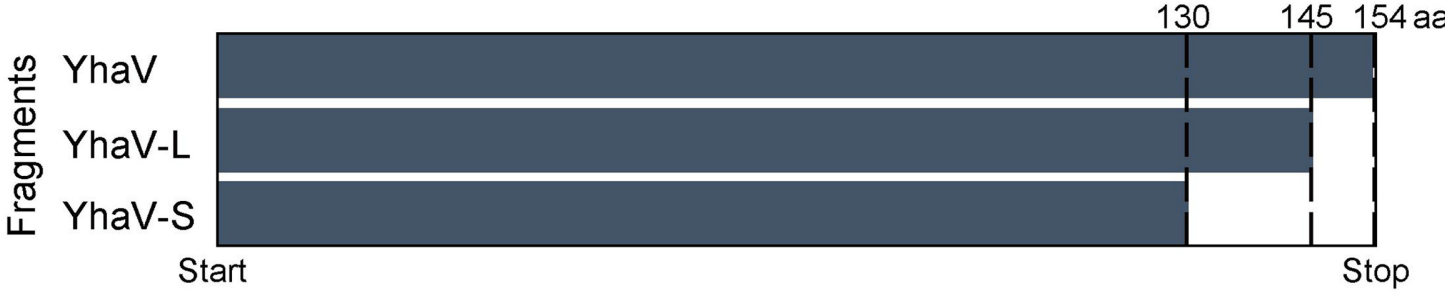

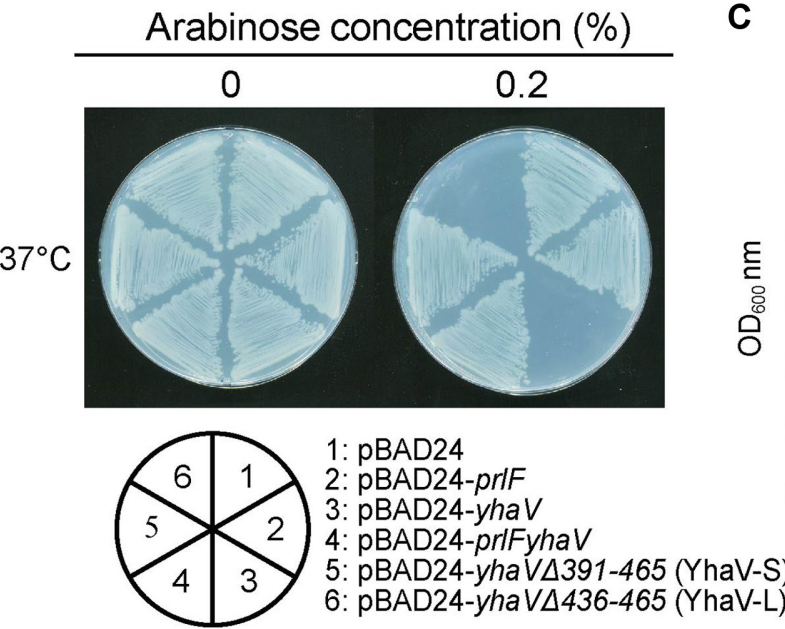

C

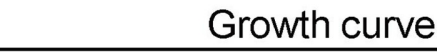

$0.2 \%$ arbinose

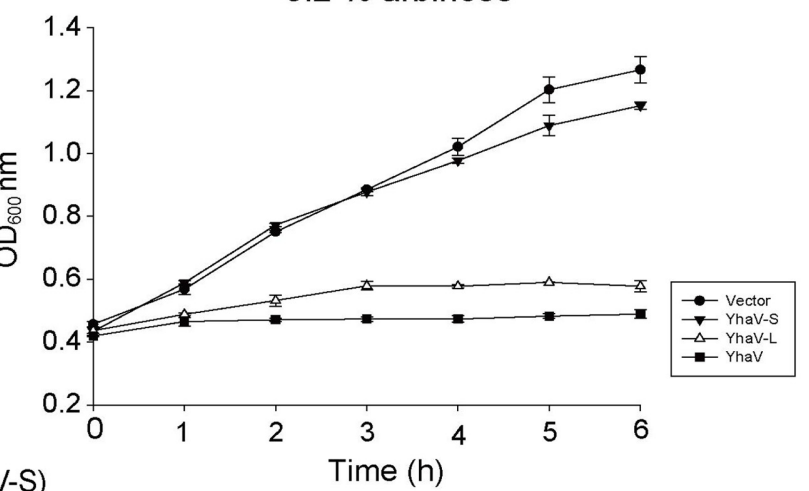

D
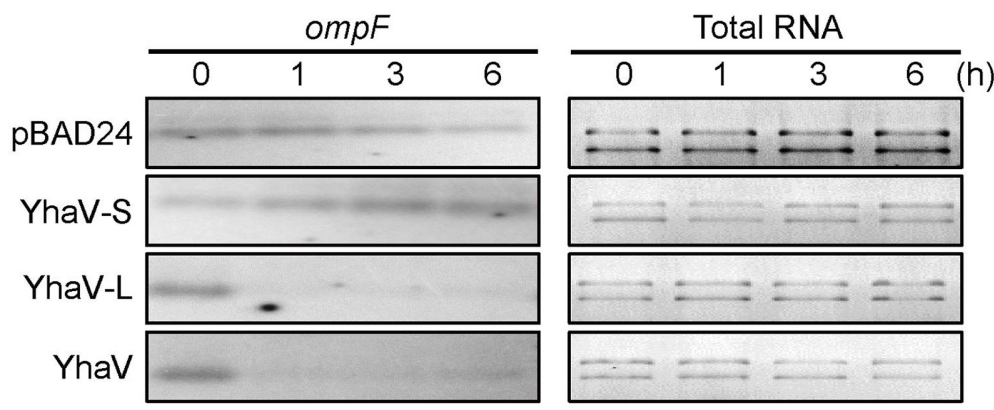

Fig. 3. Toxicity and mRNA stability analyses of YhaV and C-terminal truncated forms of YhaV.

(A) Schematic diagram of YhaV and C-terminal truncated forms of YhaVs (YhaV-L and YhaV-S). (B) Toxicity of cells harboring pBAD, pBAD24prlF, -yhaV, -prlFyhaV, -yhaV $\Delta 436-465$, or -yhaV $\Delta 391-465$ on M9-glycerol-casamino acid plates with and without L-arabinose $(0.2 \%)$ at $37^{\circ} \mathrm{C}$ for $20 \mathrm{~h}$.

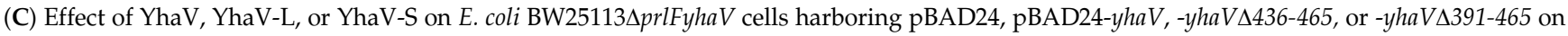
M9-glycerol-casamino acids medium at $37^{\circ} \mathrm{C}$ for $6 \mathrm{~h}$ in the presence of L-arabinose $(0.2 \%)$. Cell densities were measured for $6 \mathrm{~h}$ at $1 \mathrm{~h}$ intervals. (D) Northern blot assay was performed to identify the effects of YhaV, YhaV-L, and YhaV-S on cellular mRNA stability. Total RNA was extracted

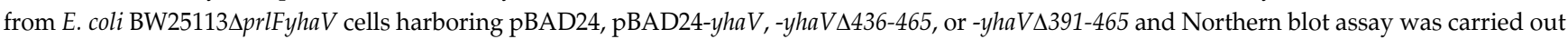
with labeled ompF. 
or absence of $0.2 \%$ arabinose. YhaV-S and smaller C-terminal truncates lost their mRNA interferase activity (Fig. S1 and Fig. 3B). These effects may be due to either conformational changes or a loss of active residues. Interestingly, YhaV $\Delta 141$ 154 was not toxic at $37^{\circ} \mathrm{C}$, was slightly toxic at $24^{\circ} \mathrm{C}$, and was toxic as full-length $\mathrm{YhaV}$ at $42^{\circ} \mathrm{C}$, indicating that toxicity is temperature sensitive (Fig. S1). To characterize the function of the C-terminal region of $Y h a V$, we chose three constructs, YhaV, YhaV-L, and YhaV-S. YhaV-S has the largest truncation and was not toxic, whereas $Y h a V$ and YhaV-L were fully toxic (Fig. 3A). We tested the ability of these constructs to inhibit growth in the presence of $0.2 \%$ arabinose at $37^{\circ} \mathrm{C}$. YhaV and the YhaV-L construct resulted in immediate growth inhibition following induction with $0.2 \%$ arabinose, whereas YhaV-S had no effect on cell growth (Fig. 3C). If the tertiary structure of the proteins encoded by these constructs is not affected, these results suggest that YhaV's C-terminal amino acid region from 131 to 154 may mediate its enzymatic activity. To evaluate the mRNA interferase activity of YhaV, YhaV-L, and YhaV-S, the stability of mRNA ompF in E. coli BW25113 $\Delta$ prlFyhaV cells harboring pBAD24-yhaV, -yhaV $4436-465$ (encoding YhaV-L), or -yhaV $\Delta 391-465$ (encoding YhaV-S) were quantified. YhaV-S did not impact $o m p F$ mRNA stability at the time intervals tested (i.e., 0, 1, 3, and $6 \mathrm{~h}$ ), whereas ompF mRNA bands were absent within $1 \mathrm{~h}$ in cells expressing YhaV and YhaV-L, confirming their mRNA interferase capacity. In addition, $16 \mathrm{~S}$ and $23 \mathrm{~S}$ ribosomal subunits were stable at all time points, suggesting that the expression of the YhaV toxin has no effect on the formation of $16 \mathrm{~S}$ and 23S ribosomes (Fig. 3D).

\section{Truncations in the C-Terminus of YhaV Did Not Affect the Secondary Structures and Thermal Stability of YhaV}

Full-length YhaV, YhaV-L, and YhaV-S were expressed in E. coli BL21(DE3) $\Delta$ prlF-yhaV cells. N-Terminally His - $^{-}$ tagged YhaV, YhaV-L, and YhaV-S were purified with NiNTA agarose (Fig. 4A). Additionally, C-terminally $\mathrm{His}_{6}$ tagged YhaV was purified with Ni-NTA agarose following the solid-phase refolding method (Fig. 4A). Using in silico approaches (i.e., ProtParam and ExPASy), the molecular masses of YhaV, YhaV-L, and YhaV-S were predicted to be $18.7,17.6$, and $15.8 \mathrm{kDa}$, respectively; the actual molecular mass of each construct was confirmed using SDS-PAGE and western blot analyses (Fig. 4).

In a previous study, the CD spectrum of YhaV renatured from the PrlF-YhaV complex by solid-phase refolding showed values as a typical helical protein that exhibits double minima at 208 and $222 \mathrm{~nm}$. To determine if C-terminal

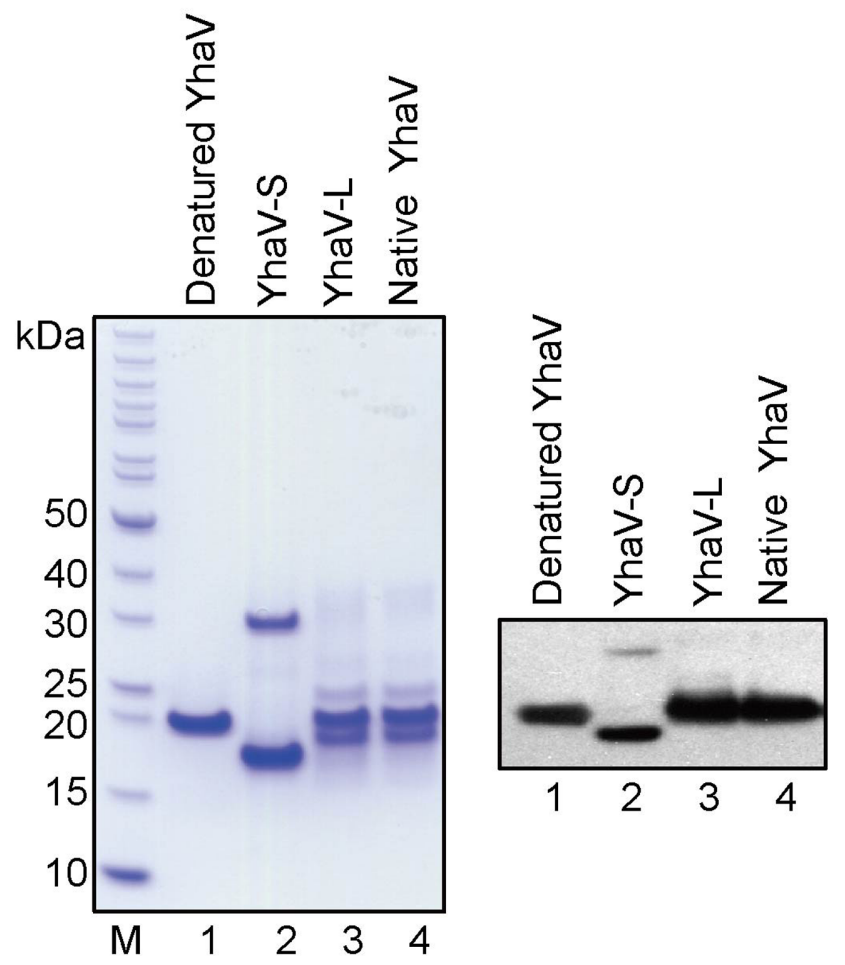

Fig. 4. Purification of native $\mathrm{YhaV}$, YhaV-L, and YhaV-S proteins.

(A) Purified denatured or native YhaV, YhaV-L, and YhaV-S on SDSPAGE. Lane 1, YhaV from pColdIII-prlFyhaV purified under denaturing conditions; lane 2, YhaV-S from pET28-yhaVA391-465 purified; lane 3, YhaV-L from pET28-yhaV 4 436-465 purified; lane 4, native YhaV from pET28-yhaV purified. (B) Western blot of denatured or native YhaV, YhaV-L, and YhaV-S probed with anti-histidine polyclonal antibody.

truncations of $\mathrm{YhaV}$ induce the destruction of the native YhaV structure or result in the loss of enzyme active residues, the secondary structures of purified YhaV, YhaV-L, and YhaV-S were compared with each other using far-UV $\mathrm{CD}$ spectrometry. The CD spectrum of purified N-terminally His $_{6}$-tagged YhaV, YhaV-L, and YhaV-S were analyzed in a wavelength range of $180-260 \mathrm{~nm}$ using a $0.5-\mathrm{mm}$ path length from $25^{\circ} \mathrm{C}$ to $85^{\circ} \mathrm{C}$ at $10^{\circ} \mathrm{C}$ intervals, and the $\mathrm{CD}$ spectra of all constructions showed no significant differences in the double minima at 208 and $222 \mathrm{~nm}$ (Figs. 5A-5C). Next, we examined how C-terminal truncations of $\mathrm{YhaV}$ affects the thermal stability of YhaV. The thermal denaturation of purified N-terminally $\mathrm{His}_{6}$-tagged $\mathrm{YhaV}$, YhaV-L, and YhaV-S were analyzed at $222 \mathrm{~nm}$ by increasing the temperature by $0.2^{\circ} \mathrm{C}$ stepwise from $25^{\circ} \mathrm{C}$ to $90^{\circ} \mathrm{C}$. The melting points of $\mathrm{YhaV}, \mathrm{YhaV}-\mathrm{L}$, and $\mathrm{YhaV}$-S were 

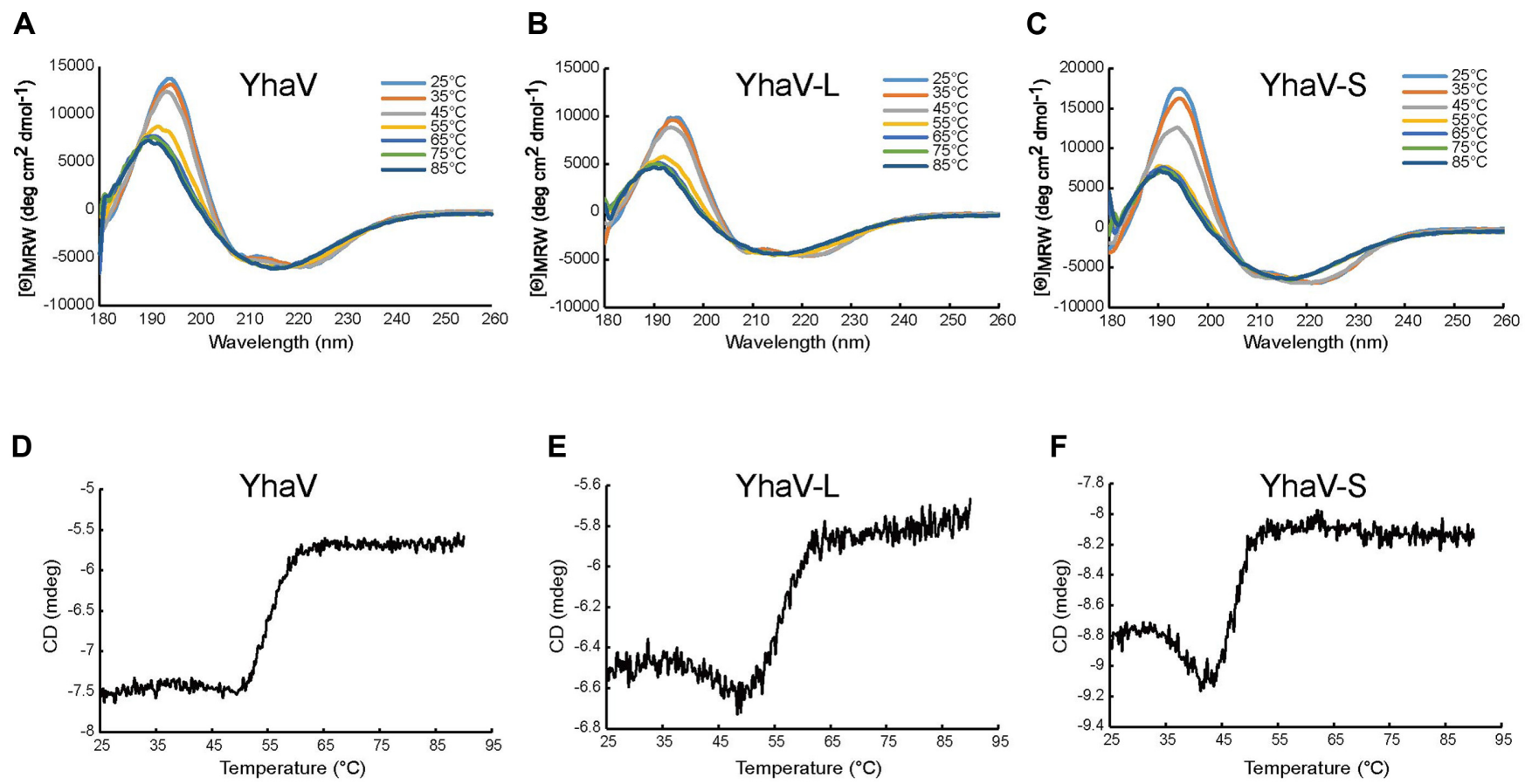

Fig. 5. Circular dichroism (CD) analysis of YhaV and C-terminal truncated forms of YhaV to confirm their secondary structures and thermostability.

(A-C) The spectra were recorded over a range of $180-260 \mathrm{~nm}$ using a $0.5-\mathrm{mm}$ path length from $25^{\circ} \mathrm{C}$ to $85^{\circ} \mathrm{C}$ at $10^{\circ} \mathrm{C}$ intervals to determine the secondary structure of YhaV, YhaV-L, and YhaV-S. (D-F) To determine the melting points of YhaV, YhaV-L, and YhaV-S, temperatures were increased by $0.2^{\circ} \mathrm{C}$ stepwise from $25^{\circ} \mathrm{C}$ to $90^{\circ} \mathrm{C}$. The background $\mathrm{CD}$ spectrum of the buffer was subtracted from the CD spectra of the protein samples.

$55^{\circ} \mathrm{C}, 48^{\circ} \mathrm{C}$, and $55^{\circ} \mathrm{C}$, respectively (Figs. 5D-5F), and the physiological stability of YhaV-L and YhaV-S were not significantly different than full-length YhaV. These results suggest that the C-terminal truncations of YhaV do not induce structural destruction of YhaV, and YhaV-S's loss of toxicity may be the result of a loss of active residues within the C-terminal region.

\section{The C-Terminal Region of YhaV Has mRNA Interferase Activity}

It has been reported that changes in cell growth and mRNA stability in vivo might be due to protein misfolding resulting from excessive protein expression [37]. Therefore, we examined the effect of full-length $Y$ haV and the C-terminal truncated forms of YhaV (YhaV-L and YhaV-S) on protein translation using a $\left.{ }^{35} \mathrm{~S}\right]$-methionine incorporation prokaryotic cell-free protein synthesis system in vitro. We measured incorporation at different concentrations (100, 500, or 1,000 nM) (Fig. 6). The PinPoint Xa vector with the chloramphenicol acetyltransferase gene (CAT) or $\beta$-lactamase gene was used in this experiment. Acetyltransferase and $\beta$ - lactamase syntheses were inhibited by purified toxin $\mathrm{YhaV}$ and YhaV-L at concentrations of 500 or 1,000 nM, whereas YhaV-S had no impact on translation at any concentration (Fig. 6). These results suggest that the C-terminal region of YhaV has mRNA interferase activity.

\section{Discussion}

mRNA interferases are enzymes that cleave RNA. These enzymes are classified into two type II classes of TA systems based on their ribosome dependency (i.e., ribosomedependent and ribosome-independent) [1]. Alignment of the amino acid sequence of $\mathrm{YhaV}$ and ribosome-dependent toxins by multiple sequence alignment suggests that $\mathrm{YhaV}$ is more closely related to ribosome-dependent toxins (e.g., RelE, YoeB, and HigB) [30-33] compared with the MazF family and MqsR, a well-known group of ribosomeindependent toxins [12, 28, 29, 36]. Moreover, the PrlFYhaV TA system in E. coli was classified as a ribosomedependent mRNA interferase with a polysome profiling assay [23]. Interestingly, there were differences in bound 


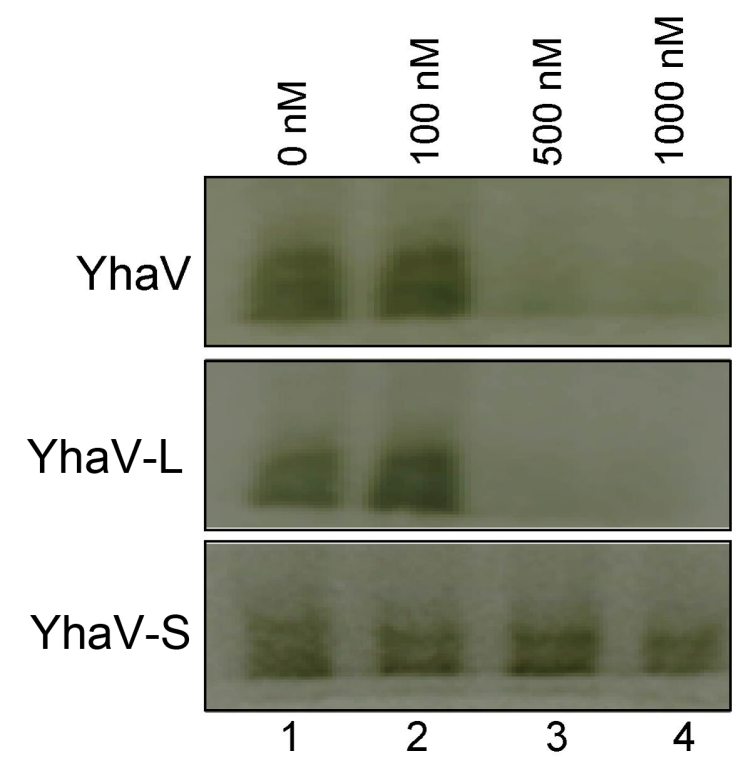

Fig. 6. Effect of C-terminal truncated forms of YhaV on protein synthesis in prokaryotic cell-free systems.

A prokaryotic cell-free protein synthesis assay was performed using different concentrations $(100,500$, and 1,000 nM) of YhaV, YhaV-L, and YhaV-S. Pellets were analyzed by SDS-PAGE followed by autoradiography.

ribosomal subunits among RelE, YoeB, and HigB when characterized by polysome profiling assays and tertiary structure models. YhaV is 154 amino acids in length, whereas RelE, YoeB, and HigB are relatively smaller in size at 95, 84, and 92 amino acids, respectively. YhaV has 20 additional residues between the first and second alphahelix when compared with secondary motifs of the RelE homolog using amino acid sequence alignments [22]. Regions similar to RelE are related mainly to the interaction with the $16 \mathrm{~S}$ ribosomal subunit, and thus the additional 20 residues in $\mathrm{YhaV}$ may also be involved in ribosomal interactions [17]. The tertiary structure of the RelE:ribosome:RNA substrate complex indicates that the Y87, R61, and R81 residues of RelE are critically involved in its enzymatic activity and that $\mathrm{K} 53$ and $\mathrm{K} 54$ might be involved in the stabilization of the negatively charged transition state. In the crystal structure of the RelE:ribosome:target RNA complex: (i) RelE binds to ribosome A sites, (ii) the aromatic ring of $\mathrm{Y} 87$ and the base ring of the second nucleotide of the target RNA were stacked, and (iii) the third nucleotide of the target RNA was stacked with the 16S ribosomal RNA. Next, Y87 of RelE induced the hydrolysis of the second and third nucleotides conveying RelE's mRNA interferase activity. H83 of another YhaV homolog,
YeoB, plays a similar role to Y87 of RelE and facilitates YeoB-mediated cleavage of target RNA [21]. In contrast, site-directed mutagenesis of Y87 to F87 did not lead to a dramatic decrease of RelE activity, perhaps because the arginines and lysines within the active sites could compensate for the role of Y87. In this study, the sequence alignments showed that aromatic residues, W143 or H154, in YhaV may play an analogous role to Y87 of RelE. However, sitedirected mutagenesis of W143 or H154 to alanine did not show the change of $\mathrm{YhaV}$ activity in toxicity test and northern blot using ompF mRNA (Fig. S2). The loss of W143 or $\mathrm{H} 154$ in YhaV might be compensated by aspartic acid, glutamic acid, or arginine within the active sites, similar to the results of site-directed mutagenesis of Y87 to F87 in RelE.

In this report, to evaluate the C-terminal function of $\mathrm{YhaV}$, we used various lengths of C-terminal truncated forms of $\mathrm{YhaV}$, rather than site-directed mutagenesis, based on the structural analysis of RelE, YeoB, and HigB homologs. Notably, in this report, we present a new lowtemperature method for the purification of native $\mathrm{YhaV}$, which was previously very difficult because of its toxicity. Furthermore, we evaluated the secondary structures and thermostability of these natively purified proteins and confirmed that there was no structural destruction, suggesting that the inhibition of cell growth in vivo was not due to structural damage. However, it has been reported that excessive protein expression may result in protein misfolding and changes in cell growth and mRNA stability. To exclude this possibility, we used an $\left[{ }^{35} \mathrm{~S}\right]$-methionine incorporation prokaryotic cell-free protein synthesis system in vitro with purified $\mathrm{YhaV}$, YhaV-L, and YhaV-S. In this way, we showed that the C-terminal region of $\mathrm{YhaV}$ is essential for mRNA interferase activity, and W143 or H154 residues might play a role similar to that of Y87 of RelE.

mRNA interferases may be of interest as (i) possible therapeutic options for the treatment of RNA virus-related diseases and (ii) secondary structural analysis of RNA since they bind specifically to single-stranded RNA and knock out rather than knock down the target gene [38]. However, for their application in knocking out specific genes, further studies are needed to improve the RNA sequence recognition length and the specificity. mRNA interferases can recognize seven nucleotides and cleave target RNAs. Similarly, zinc finger nucleases (ZFN) were developed to cleave specific DNA sequences, and those nucleases originally recognized three nucleotides. The ZFN system has advanced and can now recognize a maximum of 18 DNA nucleotides by the trimerization of three monomers and the addition of FokI, which can be dimerized and 
cleave double-stranded DNAs. Moreover, it was revealed that zinc finger protein amino acids within the $\alpha$-helix (positions $-1,+1,+2,+3,+5$, and +6 ) are the conserved amino acids for sequence recognition, and this region can be modified to acquire new sequence specificity [39]. It is likely worth developing mRNA interferases as an RNA cleavage tool similar to ZFN for DNA cleavage. For this purpose, understanding the residues mediating enzymatic activity and RNA sequence recognition might be important. It remains unknown whether the residues that are involved in target sequence recognition and cleavage are distinct or if they overlap. In a previous study, we showed the possibility of engineered mRNA interferases as a specific RNA cleavage tool by exchanging two different predicted recognition loop regions, which resulted in a chimeric protein that recognizes a new sequence and length [27]. In the case of $\mathrm{YhaV}$, important residues for the enzymatic activities are in the C-terminus like its homologs, RelE, $\mathrm{YeoB}$, and HigB. It is possible that the C-terminal region of those mRNA interferases also have target RNA sequence recognition residues. Further studies will be required to determine the tertiary structure of the YhaV:ribosome:target mRNA complex to better characterize the functions of the C-terminal region of YhaV.

\section{Acknowledgments}

We thank Dr. Munjin Kwon for critical reading of this manuscript. This work was supported by grants from the KRIBB Research Initiative Program and the NextGeneration BioGreen 21 Program (No. PJ013686012018), Rural Development Administration, Republic of Korea.

\section{Conflict of Interest}

The authors have no financial conflicts of interest to declare.

\section{References}

1. Yamaguchi Y, Park JH, Inouye M. 2011. Toxin-antitoxin systems in bacteria and archaea. Annu. Rev. Genet. 45: 61-79.

2. Pandey DP, Gerdes K. 2005. Toxin-antitoxin loci are highly abundant in free-living but lost from host-associated prokaryotes. Nucleic Acids Res. 33: 966-976.

3. Page R, Peti W. 2016. Toxin-antitoxin systems in bacterial growth arrest and persistence. Nat. Chem. Biol. 12: 208-214.

4. Thisted T, Gerdes K. 1992. Mechanism of post-segregational killing by the hok/sok system of plasmid R1. Sok antisense RNA regulates hok gene expression indirectly through the overlapping mok gene. J. Mol. Biol. 223: 41-54.

5. Kawano M, Oshima T, Kasai H, Mori H. 2002. Molecular characterization of long direct repeat (LDR) sequences expressing a stable mRNA encoding for a 35-amino-acid cell-killing peptide and a cis-encoded small antisense RNA in Escherichia coli. Mol. Microbiol. 45: 333-349.

6. Kawano M. 2012. Divergently overlapping cis-encoded antisense RNA regulating toxin-antitoxin systems from E. coli: hok/sok, ldr/rdl, symE/symR. RNA Biol. 9: 1520-1527.

7. Van Melderen L, Thi MH, Lecchi P, Gottesman S, Couturier M, Maurizi MR. 1996. ATP-dependent degradation of CcdA by Lon protease. Effects of secondary structure and heterologous subunit interactions. J. Biol. Chem. 271: 27730-27738.

8. Buts L, Lah J, Dao-Thi MH, Wyns L, Loris R. 2005. Toxinantitoxin modules as bacterial metabolic stress managers. Trends Biochem. Sci. 30: 672-679.

9. Brzozowska I, Zielenkiewicz U. 2013. Regulation of toxinantitoxin systems by proteolysis. Plasmid 70: 33-41.

10. Muthuramalingam M, White JC, Bourne CR. 2016. Toxinantitoxin modules are pliable switches activated by multiple protease pathways. Toxins (Basel) 8: E214.

11. Zhang Y, Zhang J, Hoeflich KP, Ikura M, Qing G, Inouye M. 2003. MazF cleaves cellular mRNAs specifically at ACA to block protein synthesis in Escherichia coli. Mol. Cell 12: 913-923.

12. Kamada K, Hanaoka F, Burley SK. 2003. Crystal structure of the MazE/MazF complex: molecular bases of antidote-toxin recognition. Mol. Cell 11: 875-884.

13. Yamaguchi Y, Park JH, Inouye M. 2009. MqsR, a crucial regulator for quorum sensing and biofilm formation, is a GCU-specific mRNA interferase in Escherichia coli. J. Biol. Chem. 284: 28746-28753.

14. Hwang JY, Buskirk AR. 2016. A ribosome profiling study of mRNA cleavage by the endonuclease RelE. Nucleic Acids Res. 45: 327-336.

15. Pedersen K, Zavialov AV, Pavlov MY, Elf J, Gerdes K, Ehrenberg M. 2003. The bacterial toxin RelE displays codonspecific cleavage of mRNAs in the ribosomal A site. Cell 112: 131-140.

16. Galvani C, Terry J, Ishiguro EE. 2001. Purification of the RelB and RelE proteins of Escherichia coli: RelE binds to RelB and to ribosomes. J. Bacteriol. 183: 2700-2703.

17. Neubauer C, Gao YG, Andersen KR, Dunham CM, Kelley AC, Hentschel J, et al. 2009. The structural basis for mRNA recognition and cleavage by the ribosome-dependent endonuclease RelE. Cell 139: 1084-1095.

18. Zhang Y, Inouye M. 2009. The inhibitory mechanism of protein synthesis by YoeB, an Escherichia coli toxin. J. Biol. Chem. 284: 6627-6638.

19. Keren I, Shah D, Spoering A, Kaldalu N, Lewis K. 2004. Specialized persister cells and the mechanism of multidrug tolerance in Escherichia coli. J. Bacteriol. 186: 8172-8180.

20. Zhang Y, Yamaguchi Y, Inouye M. 2009. Characterization of YafO, an Escherichia coli toxin. J. Biol. Chem. 284: 25522-25531. 
21. Feng S, Chen Y, Kamada K, Wang H, Tang K, Wang M, et al. 2013. YoeB-ribosome structure: a canonical RNase that requires the ribosome for its specific activity. Nucleic Acids Res. 41: 9549-9556.

22. Schmidt O, Schuenemann VJ, Hand NJ, Silhavy TJ, Martin J, Lupas AN, et al. 2007. prlF and yhaV encode a new toxinantitoxin system in Escherichia coli. J. Mol. Biol. 372: 894-905.

23. Choi W, Yamaguchi Y, Lee JW, Jang KM, Inouye M, Kim SG, et al. 2017. Translation-dependent mRNA cleavage by YhaV in Escherichia coli. FEBS Lett. 591: 1853-1861.

24. Griffin MA, Davis JH, Strobel SA. 2013. Bacterial toxin RelE: a highly efficient ribonuclease with exquisite substrate specificity using atypical catalytic residues. Biochemistry 52: 8633-8642.

25. Shinohara M, Guo JX, Mori M, Nakashima T, Takagi H, Nishimoto E, et al. 2010. The structural mechanism of the inhibition of archaeal RelE toxin by its cognate RelB antitoxin. Biochem. Biophys. Res. Commun. 400: 346-351.

26. Baker KE, Mackie GA. 2003. Ectopic RNase E sites promote bypass of 5'-end-dependent mRNA decay in Escherichia coli. Mol. Microbiol. 47: 75-88.

27. Park JH, Yoshizumi S, Yamaguchi $\mathrm{Y}, \mathrm{Wu} \mathrm{KP}$, Inouye $\mathrm{M}$. 2013. ACA-specific RNA sequence recognition is acquired via the loop 2 region of MazF mRNA interferase. Proteins 81: 874-883.

28. Simanshu DK, Yamaguchi Y, Park JH, Inouye M, Patel DJ. 2013. Structural basis of mRNA recognition and cleavage by toxin MazF and its regulation by antitoxin MazE in Bacillus subtilis. Mol. Cell 52: 447-458.

29. Kamphuis MB, Bonvin AM, Monti MC, Lemonnier M, Munoz-Gomez A, van den Heuvel RH, et al. 2006. Model for RNA binding and the catalytic site of the RNase Kid of the bacterial parD toxin-antitoxin system. J. Mol. Biol. 357:
115-126.

30. Boggild A, Sofos N, Andersen KR, Feddersen A, Easter AD, Passmore LA, et al. 2012. The crystal structure of the intact E. coli RelBE toxin-antitoxin complex provides the structural basis for conditional cooperativity. Structure 20: 1641-1648.

31. Kamada K, Hanaoka F. 2005. Conformational change in the catalytic site of the ribonuclease YoeB toxin by YefM antitoxin. Mol. Cell 19: 497-509.

32. Schureck MA, Dunkle JA, Maehigashi T, Miles SJ, Dunham CM. 2015. Defining the mRNA recognition signature of a bacterial toxin protein. Proc. Natl. Acad. Sci. USA 112: 13862-13867.

33. Schureck MA, Repack A, Miles SJ, Marquez J, Dunham CM. 2016. Mechanism of endonuclease cleavage by the HigB toxin. Nucleic Acids Res. 44: 7944-7953.

34. Guex N, Peitsch MC. 1997. SWISS-MODEL and the SwissPdbViewer: an environment for comparative protein modeling. Electrophoresis 18: 2714-2723.

35. Herraez A. 2006. Biomolecules in the computer: Jmol to the rescue. Biochem. Mol. Biol. Educ 34: 255-261.

36. Brown BL, Grigoriu S, Kim Y, Arruda JM, Davenport A, Wood TK, et al. 2009. Three dimensional structure of the MqsR:MqsA complex: a novel TA pair comprised of a toxin homologous to RelE and an antitoxin with unique properties. PLoS Pathog. 5: e1000706.

37. Selkoe DJ. 2003. Folding proteins in fatal ways. Nature 426: 900-904.

38. Park JH, Yamaguchi Y, Inouye M. 2012. Intramolecular regulation of the sequence-specific mRNA interferase activity of MazF fused to a MazE fragment with a linker cleavable by specific proteases. Appl. Environ. Microbiol. 78: 3794-3799.

39. Chandrasegaran S, Carroll D. 2016. Origins of programmable nucleases for genome engineering. J. Mol. Biol. 428: 963-989. 